\title{
The energization of relativistic electrons in the outer Van Allen radiation belt
}

\author{
YUE CHEN*, GEOFFREY D. REEVES AND REINER H. W. FRIEDEL \\ Space Science and Applications (ISR-1 Group), Los Alamos National Laboratory, P0 Box 1663, Los Alamos, New Mexico 87545, USA \\ *e-mail: cheny@lanl.gov
}

The origin and dynamics of the Van Allen radiation belts is one of the longest-standing questions of the space age, and one that is increasingly important for space applications as satellite systems become more sophisticated, smaller and more susceptible to radiation effects. The precise mechanism by which the Earth's magnetosphere is able to accelerate electrons from thermal to ultrarelativistic energies $(E \gg 0.5 \mathrm{MeV})$ has been particularly difficult to definitively resolve. The traditional explanation is that large-scale, fluctuating electric and magnetic fields energize particles through radial diffusion ${ }^{1}$. More recent theories ${ }^{2,3}$ and observations ${ }^{4,5}$ have suggested that gyro-resonant waveparticle interactions may be comparable to or more important than radial diffusion. Using data collected simultaneously by multiple satellites passing through the magnetosphere at different distances from the Earth, we demonstrate that the latter of these is the dominant mechanism responsible for relativistic electron acceleration. Specifically, we identify frequent and persistent peaks in equatorial electron phase space density near or inside geosynchronous orbit that provide unambiguous evidence for local wave-particle acceleration. These observations represent an important step towards a more complete physical understanding of radiation belt dynamics and to the development of space-weather models.

Before the mid-1990s the proximate source of the radiation belts was thought to be electrons in the Earth's plasma sheet (a region in the Earth's magnetotail, extending from approximately seven Earth radii, $R_{\mathrm{E}}$, to many hundreds of $R_{\mathrm{E}}$ in the antisunward direction). Fluctuations in the magnetospheric electric and magnetic fields produce radial diffusion that will transport electrons from the plasma sheet towards the Earth, where the loss rates due to scattering into the atmosphere exceed diffusion rates. The high scattering loss rates near the Earth $\left(\lesssim 2.5 R_{\mathrm{E}}\right)$ act as a sink, forming the so-called slot region. As electrons diffuse from the high-altitude source to the low-altitude sink they gain energy through betatron/Fermi acceleration ${ }^{1}$ as they move to regions of higher magnetic field strength. Two characteristics of diffusive betatron/Fermi acceleration in the inner magnetosphere are that particles gain more kinetic energy in the component perpendicular to the magnetic field and that the electron phasespace density (PSD, the electron flux divided by the square of the momentum) evolves towards a monotonic radial gradient from source to sink. Spacecraft observations were generally consistent with this theoretical framework until multisatellite studies probed the structure and dynamics of the radiation belts with greater spatial and temporal resolution.

Studies using NASA's Polar satellite, along with ongoing observations from geosynchronous and Global Positioning System
(GPS) constellations, were among the first of the multispacecraft investigations of the radiation belts. In particular, observations during a large geomagnetic storm in January 1997 (ref. 6) showed flux enhancements deep in the radiation belts that were more rapid than expected, as well as evidence of outward radial diffusion from the inner magnetosphere towards the plasma sheet. These and subsequent observations stimulated development of a variety of alternative theories for relativistic electron acceleration ${ }^{7}$. Leading mechanisms include coherent drift-resonant interactions with ultralow-frequency waves ${ }^{8}$ and gyro-resonant interactions with very-low-frequency (VLF) waves ${ }^{2,3}$.

Electrons in the geomagnetic field undergo three periodic motions: gyration, bounce and gradient/curvature drift ${ }^{9}$. Each motion is associated with an adiabatic invariant and a characteristic timescale. The conservation of all three invariants leads to constant motion along a fixed 'drift shell' defined by the quantity $L^{*}$. (The Roederer $L$-parameter ${ }^{9}, L^{*}$, is a function of the third adiabatic invariant and measures the equatorial radial distance (in $R_{\mathrm{E}}$ ) of a drift shell from the Earth's centre when the drift shell is adiabatically mapped back into a dipolar magnetic field.) Although flux is not necessarily a conserved quantity of the particle motion, PSD is conserved if the adiabatic invariants are conserved. During dynamic changes, we use deviations from conservation of PSD to investigate which adiabatic invariants are violated. Fluctuations associated with radial diffusion have timescales greater than a few seconds and can violate only the third, or drift, invariant. VLF waves, with frequencies of kilohertz, will break all three invariants and can produce energy and pitch-angle diffusion in addition to radial diffusion. Because of the steeply falling electron energy spectrum, energy diffusion will result in enhancement of energetic electron fluxes (and PSD) at the location of the waves. Therefore, whereas acceleration driven by inward radial diffusion arises from PSD profiles that increase monotonically with radius (Fig. 1a), energy diffusion can produce radial profiles with a local peak of PSD (Fig. 1b). Striking evidence that this topic remains controversial is seen in two recent papers that both examined a large radiation-belt event known as the Halloween 2003 storm. One study concluded that the electron enhancement was caused by local acceleration from VLF chorus ${ }^{5}$ and the other concluded that the enhancement was caused by enhanced diffusion from ultralowfrequency drift resonance ${ }^{10}$.

The first step in distinguishing between acceleration by radial diffusion and acceleration by gyro-resonant wave-particle interactions is to convert from spacecraft observations of flux at fixed energy to PSD at fixed values of the adiabatic invariants ${ }^{11}$ and then examine the radial gradients. Polar observations showed the first evidence for a localized, radial peak in PSD that developed 

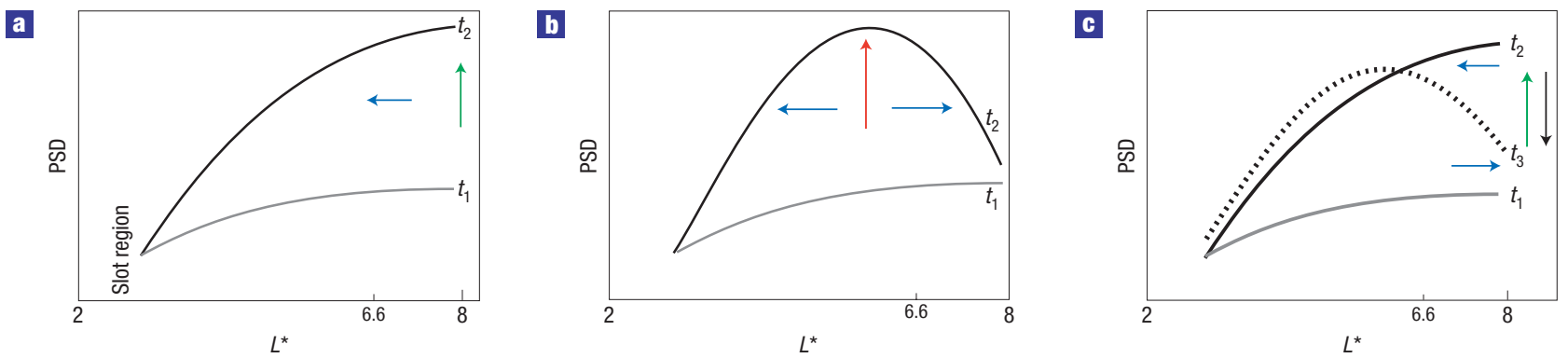

Figure 1 The shape of equatorial PSD profiles as a function of $L^{*}$ differentiates between diffusive and local acceleration processes. Here $L^{*}$ approximately indicates the radial distance of the drift shell in Earth radii $\left(R_{\mathrm{E}}\right)$. The grey curve in each panel (labelled $\left.t_{1}\right)$ represents the PSD profile before acceleration and the black curve (labelled $t_{2}$ ) is the profile after acceleration. The 'slot region' at low $L^{*}$ is a sink, where interaction with VLF hiss scatters electrons into the atmosphere. a, Inward radial diffusion (the blue arrow) from an increasing plasma-sheet source population (green arrow) produces gradients that increase monotonically with radius. In particular, the gradient in the vicinity of geosynchronous orbit (at $6.6 R_{\mathrm{E}}$ ) should always be positive. $\mathbf{b}$, Local acceleration (red arrow) should characteristically produce radial peaks in PSD with negative gradients outside geosynchronous orbit. Subsequent diffusion (blue arrows) will gradually flatten these peaks. c, Local PSD peaks can also be produced by a plasma-sheet source population that first increases (green arrow at $t_{2}$ ) and then decreases (black arrow at $t_{3}$ ). In this scenario, peaks should be most common during times between acceleration events.
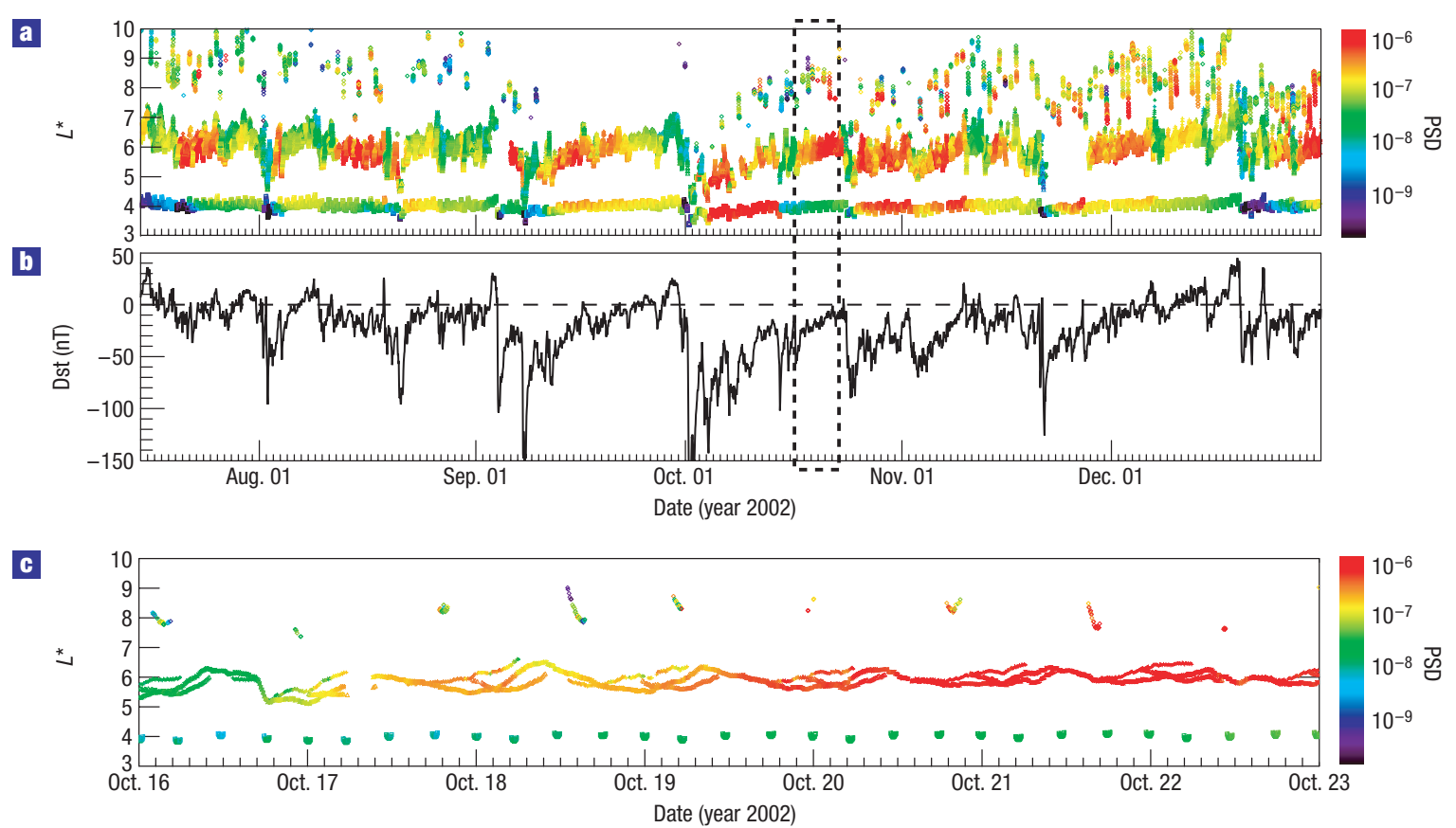

Figure 2 Equatorial PSD as a function of $L^{*}$ (radial drift shell) and time compared with magnetic activity (Dst). a,b, PSD distributions and magnetic activity (Dst) during a $\sim 6$ month period. The colour-coded PSD data points are from GPS (at $L^{*} \approx 4$ ), LANL GE0 (medium $L$ shells) and Polar (highest $L$ shells), with first invariant $\mu=2,083 \mathrm{MeV} \mathrm{G}^{-1}$ and second invariant $K=0.03 \mathrm{G}^{1 / 2} R_{\mathrm{E}}$. The unit of PSD is $\left(\mathrm{c}^{3} \mathrm{MeV}^{-3} \mathrm{~cm}^{-3}\right)$. Negative values of Dst are a standard index of geomagnetic storm strength. c, PSD as a function of $L^{*}$ and time for October 16-22 (the time period boxed in a and $\mathbf{b}$ ) showing in detail the rise of geosynchronous PSDs before any increases at lower $L^{*}$ (GPS) or higher $L^{*}$ (Polar).

during the main phase of a moderate geomagnetic storm in April 1998 (ref. 4). These observations did not definitively resolve the controversy, however, because the high latitude of the Polar observations prevented determination of PSDs for electrons with equatorial pitch angles $\alpha_{\text {equ }} \gtrsim 35^{\circ}$. It is possible to have a radial peak in PSD at small pitch angles measured well away from the magnetic equator but still with a monotonic radial gradient in PSD at the equator. This situation would occur if the primary acceleration were produced by radial diffusion (producing the monotonic gradient) but pitch-angle scattering were stronger at some radial distances than at others, which would make more electrons observable off the equator in a limited region of $L^{*}$, even though they are not preferentially accelerated there.

Very recent re-examination of data from the NASA/USAF Combined Release and Radiation Effects Satellite (CRRES) in geosynchronous transfer orbit confirmed the Polar observations of peaked profiles of PSD using near-equatorial observations of three storms, but these observations were limited in energy $(E<600 \mathrm{keV}$ 

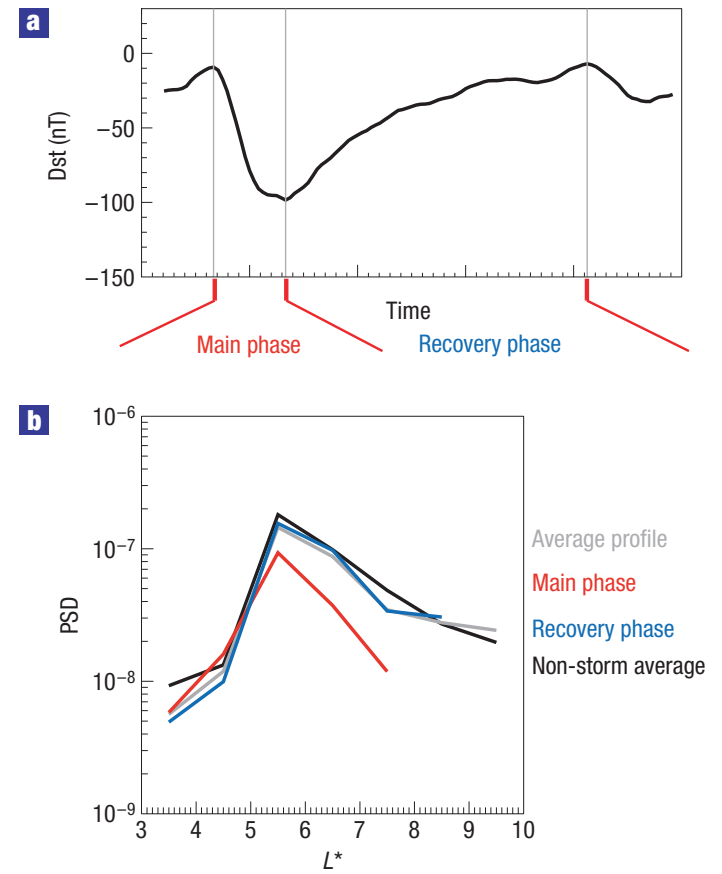

Figure 3 A superposed epoch analysis that bins PSD as a function of $L^{*}$ and geomagnetic storm phase during a two year period (2001-2002). Here the first invariant $\mu=2,083 \mathrm{MeV} \mathrm{G}^{-1}$, the second invariant $K=0.03 G^{1 / 2} R_{\mathrm{E}}$ and the unit of $\mathrm{PSD}$ is $\left(\mathrm{c}^{3} \mathrm{MeV}^{-3} \mathrm{~cm}^{-3}\right)$. a, Each geomagnetic storm is divided into the main phase, when the Dst index is becoming more negative, and the recovery phase, when Dst is returning toward zero. Minimum Dst is taken as the zero time for this superposed epoch study. $\mathbf{b}$, The radial PSD distributions for all geomagnetic conditions averaged over available data from the two year period 2001-2002 (grey), during storm main phases (red), during storm recovery phases (blue) and during times when Dst indicated no geomagnetic storm activity (black). Here $L^{*}$ has a bin size of 1 and the central-point value of the first bin is 3.5 .

at $\left.L^{*}=6\right)$, spatial coverage $\left(L^{*}<6\right)$ and time resolution (one orbit $\approx 10 \mathrm{~h})^{12}$. That study left open the possibility that PSD peaks formed as a result of a varying source population in the plasma sheet $\left(L^{*}>6\right)$. As the plasma-sheet population at large $L^{*}$ varies, diffusion acts to move particles from regions of high PSD to lower. If the timescale of variation of the source is fast compared with diffusion then short-lived peaks in PSD can again be produced without local acceleration ${ }^{4}$ (Fig. 1c).

To overcome previous ambiguities we investigated six months of simultaneous observations of equatorial PSD obtained from multiple satellites at a variety of radial distances: a GPS satellite with equatorial crossings at $R=4.2 R_{\mathrm{E}}$, geosynchronous satellites ${ }^{13,14}$ at $R=6.6 R_{\mathrm{E}}$ and Polar ${ }^{15}$ at $R>7 R_{\mathrm{E}}$. Following the method of ref. 16 , we used the Tsyganenko 2001 storm-time magnetic field model ${ }^{17}$ (T01s), along with the measured electron fluxes, to calculate the PSD for each measurement at fixed values of the first and second invariants, $\mu=2,083 \mathrm{MeV} \mathrm{G}^{-1}$ and $K=0.03 G^{1 / 2} R_{\mathrm{E}}$. These values correspond to relativistic electron energies $(E \gtrsim 1 \mathrm{MeV})$ and near-equatorial pitch angles $\left(\alpha_{\text {equ }} \sim 40-80^{\circ}\right)$. As radial diffusion enhances the velocity component perpendicular to the magnetic field, the resulting energization is strongest when observed near the magnetic equator. Therefore, equatorial measurements provide the key observations that were not previously investigated in this region of space $e^{4,11,12}$.

In an asymmetric and time-variable magnetic field, spacecraft at a fixed radial distance (such as geosynchronous satellites) will measure a range of magnetic drift shells that encompass varying amounts of magnetic flux, the conserved third invariant. This effect is particularly strong during magnetic storms, when diamagnetic currents inflate the drift shells, creating large, but fully adiabatic, changes in observed electron flux ${ }^{18}$. As was the case for acquiring equatorial measurements, accurate determination of radial PSD profiles requires accounting for adiabatic changes due to the global magnetic field. Here we used a specifically developed storm-time field model and tested our calculations of PSD by comparing simultaneous geosynchronous measurements obtained at different local times (see the Methods section).

Once these requirements have been met, it becomes readily apparent that peaks in the radial profile of PSD are a common, and even typical, response to geomagnetic activity. In Fig. 2a the near-equatorial PSD as a function of $L^{*}$ and time for three geosynchronous satellites, one GPS satellite, and Polar show that the geosynchronous PSDs (at $\left.L^{*} \approx 6\right)$ are frequently higher than both those measured at GPS $\left(L^{*} \approx 4\right)$ and Polar $\left(L^{*}>6\right)$. We note that a maximum value of PSD observed at geosynchronous orbit does not necessarily mean that the acceleration region is exactly collocated as electrons accelerated at a different $L^{*}$ will subsequently diffuse from the peak towards geosynchronous orbit if the gradient is in the proper direction.

The largest changes in PSD and the strongest relative peaks (Fig. 2a) are observed during periods of enhanced geomagnetic activity as indicated by negative dips of the Dst index ${ }^{19}$ (Fig. 2b). Figure $2 \mathrm{c}$ demonstrates this in greater detail for the moderate storm starting on 16 October 2002. On 17 October the geosynchronous PSDs begin to rise above prestorm levels, while the Polar and GPS PSDs remain essentially unchanged. Geosynchronous PSDs continue to rise, peaking around 21 October. In contrast, Polar PSDs actually decrease during the orbital pass on 18 October before increasing above prestorm levels on subsequent orbits. This particular storm apparently did not substantially affect the radiation belts at GPS altitudes $\left(L^{*} \approx 4\right)$-which is not the case for all storms (Fig. 2a).

We emphasize the relative timing of the rises and decreases of PSD at different $L^{*}$ because of its importance in distinguishing between local acceleration (Fig. 1b) and the combined effects of a variable source plus losses at high $L^{*}$ (Fig. 1c). For a variable plasma-sheet source to produce radial peaks, it must first produce an increase at high $L^{*}$ and a monotonic radial gradient, followed by decreases at the outermost $L^{*}$, which is contrary to the observations shown here.

We have carried out the same analysis for energies that give $\mu$ values from 462 to $2,083 \mathrm{MeVG}^{-1}$ and found the same general behaviour. At lower energies, we expect the dynamics of the electrons to become increasingly affected by other processes, such as $\mathbf{E} \times \mathbf{B}$ drift, and these results should not be generalized to $\mu \lesssim 460 \mathrm{MeVG}^{-1}$. Although we know that electrons in the radiation belts can be accelerated to ultrarelativistic energies (for example $E>10 \mathrm{MeV}$ (ref. 20)), in this study we use $\mu$ values that correspond to $E \lesssim 1 \mathrm{MeV}$ at Polar, $E \lesssim 1.5 \mathrm{MeV}$ at geosynchronous orbit and $E \lesssim 4 \mathrm{MeV}$ at GPS. We do not know whether the results here can be generalized to higher energies.

We have also extended this analysis to a full two years using the available observations during 2001 and 2002. We binned all observations over these two years according to drift shell $\left(L^{*}\right)$ and geomagnetic storm phase (Fig. 3). The main phase of a geomagnetic storm is defined as the interval when the Dst index is decreasing towards its minimum value (maximum storm intensity), and the recovery phase is the interval when the Dst index returns towards values approaching zero (geomagnetic quiet), as shown in Fig. 3a. We compared the average PSD radial profile (Fig. 3b) for the full two year period regardless of geomagnetic 
activity, for the subset of storm main phases, for storm recovery phases, and for non-storm times (that is, all times not identified as main or recovery phases).

The superposed epoch analysis shows that PSD peaks in the vicinity of $L^{*} \approx 5-6$ are a common feature of the outer electron belt and not an occasional or rare occurrence (Fig. 3b). Although there is clear variation in PSD with geomagnetic activity (Fig. 2), the average PSD profiles during the main, recovery and nonstorm phases are quite similar. The PSD increases at intermediate distances (seen first here at the synchronous satellites) during the storm main phase or early recovery phase (Fig. 2). Once the peak is formed, the electrons are gradually redistributed through radial diffusion but persist for times longer than the storm recovery times, and hence are present even in non-storm times. It is interesting to note that the PSDs at $L^{*} \gtrsim 5$ are generally lower during storm main phases, when electron losses are the strongest ${ }^{2,7,21}$, than at other times (Fig. 3b).

These observations, we believe, conclusively demonstrate that local acceleration is the dominant mechanism for relativistic electron acceleration in the radiation belts. Local acceleration implies interactions on timescales of the electron gyro-period and therefore must involve waves in the VLF frequency range. Five possible wave modes ${ }^{2}$ could potentially produce the acceleration characteristics that we have identified. Although the effectiveness of electron acceleration by each wave mode has not yet been examined in detail, interaction with whistler mode VLF chorus is now considered the most likely candidate for electron acceleration ${ }^{2,3}$. Recent observations ${ }^{5}$ have shown that VLF chorus is indeed observed at the right phase of a storm, in association with an extremely strong radiation-belt enhancement event, providing evidence that is in concert with the prediction of this theory.

Although multisatellite observations of PSD peaks help pinpoint the nature of the dominant acceleration processes and regions, they leave significant unanswered questions regarding the precise physical mechanisms for acceleration and losses and the solar wind drivers that ultimately provide the free energy for these processes.

\section{METHODS}

The differential electron-flux data are from the measurements of the Los Alamos GPS energetic particle sensor (BDD-IIR), LANL GEO Synchronous Orbit Particle Analysis (SOPA) instruments ${ }^{14}$ and Polar's Comprehensive Energetic Particle and Pitch Angle Distribution (CEPPAD) experiment ${ }^{15}$. A more detailed description of the data and the method of transforming fluxes to PSD distributions are outlined elsewhere ${ }^{16,22}$. For a study of PSD distributions from multisatellite observations, errors are mainly introduced from two sources: one is instrument intercalibration, which can systematically change the relative values of PSD from one satellite to another, and the other is the imperfect magnetospheric magnetic-field model, which will put a PSD point at the wrong phase-space coordinates. Here special measures have been taken to constrain and estimate errors from both sources. For instrument intercalibration, a two-step procedure is followed: first, continuous energy spectra are obtained from the instrument count rates and energy-dependent response functions during times when two satellites are in magnetic conjunction to obtain improved calibration factors for each instrument ${ }^{23}$; then, after PSD distributions are calculated, the PSD values with the same phase-space coordinates of $\left(\mu, K, L^{*}\right)$ but measured by spacecraft at different spatial locations are again compared during magnetic quiet times to verify and adjust the intercalibration factors ${ }^{22}$. For the magnetic field, we chose the T01s model from a list of models (including the Tsyganenko 1996 model (T96; refs 24,25$)$ ) because the T01s statistically has the best performance of fitting to in situ magnetic-field measurements, especially during storm times ${ }^{22}$. To test the dependence on the magnetic-field model, we repeated the calculation of PSD distributions during the same period shown in Fig. 2 and found that the PSD radial distributions stay overall unchanged (see Fig. A1 in the Supplementary Information). Additionally, as the PSD radial shape heavily depends on the calculation of the second phase-space coordinate $K$, we also study the dependence of PSD values on $K$, as depicted by Fig. A2 in the Supplementary Information, and conclude that potential errors in the calculation of $K$ change the absolute PSD values but continue to produce PSDs that peak near geosynchronous orbit.

Received 30 January 2007; accepted 4 June 2007; published 1 July 2007.

\section{References}

1. Schulz, M. \& Lanzerotti, L. Particle Diffusion in the Radiation Belts (Springer, New York, 1974)

2. Horne, R. B. \& Thorne, R. M. Potential waves for relativistic electron scattering and stochastic acceleration during magnetic storms. Geophys. Res. Lett. 25, 3011-3014 (1998).

3. Summers, D., Thorne, R. M. \& Xiao, F. Relativistic theory of wave-particle resonant diffusion with application to electron acceleration in the magnetosphere. J. Geophys. Res. 103, 20487-20500 (1998).

4. Green, J. C. \& Kivelson, M. G. Relativistic electrons in the outer radiation belt: Differentiating between acceleration mechanisms. J. Geophys. Res. 109, A03213 (2004).

5. Horne, R. B. et al. Wave acceleration of electrons in the Van Allen radiation belts. Nature 437, 227-230 (2005)

6. Reeves, G. D. et al. The global response of relativistic radiation belt electrons to the January 1997 magnetic cloud. Geophys. Res. Lett. 25, 3265-3268 (1998).

7. Friedel, R., Reeves, G. \& Obara, T. Relativistic electron dynamics in the inner magnetosphere-A review. J. Atmos. Sol. Terr. Phys. 64, 265-282 (2002).

8. Elkington, S. R., Hudson, M. K. \& Chan, A. A. Acceleration of relativistic electrons via drift resonant interactions with toroidal-mode Pc-5 ULF oscillations. Geophys. Res. Lett. 26, 3273-3276 (1999).

9. Roederer, J. G. Dynamics of Geomagnetically Trapped Radiation (Springer, New York, 1970).

10. Loto'aniu, T. M. et al. Radial diffusion of relativistic electrons into the radiation belt slot region during the 2003 Halloween geomagnetic storms. J. Geophys. Res. 111, A04218 (2006).

11. Taylor, M. G. G. T. et al. Multisatellite measurements of electron phase space density gradients in the Earth's inner and outer magnetosphere. J. Geophys. Res. 109, A05220 (2004).

12. Iles, R. H. A., Meredith, N. P., Fazakerley, A. N. \& Horne, R. B. Phase space density analysis of the outer radiation belt energetic electron dynamics. J. Geophys. Res. 111, A03204 (2006).

13. Bame, S. J. et al. Magnetospheric plasma analyzer for spacecraft with constrained resources. Rev. Sci. Instrum. 64, 1026-1033 (1993).

14. Belian, R., Gisler, G., Cayton, T. \& Christensen, R. High- $z$ energetic particles at geosynchronous orbit during the great solar proton event series of October 1989. J. Geophys. Res. 97, 16897-16906 (1992).

15. Blake, J. B. et al. CEPPAD: Comprehensive energetic particle and pitch angle distribution experiment on Polar. Space Sci. Rev. 71, 531-562 (1995).

16. Chen, Y., Friedel, R. H. W., Reeves, G. D., Onsager, T. G. \& Thomsen, M. F. Multisatellite determination of the relativistic electron phase space density at geosynchronous orbit: Methodology and results during geomagnetically quiet times. J. Geophys. Res. 110, A10210 (2005).

17. Tsyganenko, M., Singer, H. \& Kasper, J. Storm-time distortion of the inner magnetosphere: how severe can it get? J. Geophys. Res. 108, 1209 (2003).

18. Kim, H. \& Chan, A. Fully-adiabatic changes in storm-time relativistic electron fluxes. J. Geophys. Res. $102,22107-22116(1997)$.

19. Iyemori, T., Maeda, H. \& Kamei, T. Impulse response of geomagnetic indices to interplanetary magnetic field. J. Geomagn. Geoelectr. 31, 1-9 (1979).

20. Blake, J. B., Kolasinski, W. A., Fillius, R. W. \& Mullen, E. G. Injection of electrons and protons with energies of tens of MeV into $L<3$ on 24 March 1991. Geophys. Res. Lett. 19, 821-824 (1992).

21. Reeves, G. D., McAdams, K. L., Friedel, R. H. W. \& O'Brien, T. P. Acceleration and loss of relativistic electrons during geomagnetic storms. Geophys. Res. Lett. 30, 1529 (2003).

22. Chen, Y., Friedel, R. H. W. \& Reeves, G. D. Phase space density distribution of energetic electrons in the outer radiation belt during two Geospace Environment Modeling Inner Magnetosphere/Storms selected storms. J. Geophys. Res. 111, A11S04 (2006)

23. Friedel, R. H. W., Bourdarie, S. \& Cayton, T. Intercalibration of magnetospheric energetic electron data. Space Weather 3, S09B04 (2005).

24. Tsyganenko, M. Modeling the Earth's magnetospheric magnetic field confined within a realistic magnetopause. J. Geophys. Res. 100, 5599-5621 (1995).

25. Tsyganenko, M., Singer, H. \& Kasper, J. Storm-time distortion of the inner magnetosphere: How severe can it get? J. Geophys. Res. 101, 27187-27198 (1996).

\section{Acknowledgements}

This work was supported by the National Science Foundation's Geospace Environment Modeling Program, the NASA Polar satellite program and the US Department of Energy. We thank T. Cayton and R. Christsen for providing LANL GEO SOPA and GPS BDD-IIR data. We are grateful for the use and R. Christsen for providing LANL GEO SOPA and GPS BDD-IIR data. We are grateful for the use of ONERA-DESP codes provided by D. Boscher
OMNIWeb for the use of omni solar wind data.

Correspondence and requests for materials should be addressed to Y.C.

Supplementary Information accompanies this paper on www.nature.com/naturephysics.

\section{Author contributions}

All co-authors contributed in the form of data generation and/or discussion and critical comment.

\section{Competing financial interests}

The authors declare no competing financial interests.

Reprints and permission information is available online at http://npg.nature.com/reprintsandpermissions/ 